\section{P285 FACTORS ASSOCIATED WITH MOTHER-TO-CHILD HIV TRANSMISSION IN WESTERN NIGERIA: IMPORTANCE OF 90-90-90 GOALS IN THE ELIMINATION}

Saheed Usman*, Prosper Okonkwo, Oluwatoyin Jolayemi, Jay Osi-Samuels, Patrick Akande, Babatunde Ladi-Akinyemi, Oluremi Olaitan, Femi Owolagba, Matthias Alagi, Eke Ofuche. APIN Public Health Intiatives, Abuja, Nigeria

\subsection{6/sextrans-2019-sti.408}

Background HIV pandemic has continued to be a huge challenge in Nigeria, with the problem of stigmatization reducing the chances of early determination of the HIV status of pregnant women. Hypotheses tested were the influence of maternal antiretroviral therapy (ART) use and infant's feeding option on baby's final early infant diagnosis (EID) outcome. The study was aimed at evaluating the variables associated with mother-to-child transmission of HIV \& the factors associated with the transmission of the infection from the mothers. Methods This study was a prospective cohort study of HIVexposed infants conducted in Western Nigeria, between January 2015 and September 2017. Dried Blood Spots (DBS) were analysed using polymerase chain reaction technique. All data collected using Epidata and were statistically analysed, using statistical package for the social sciences (SPSS).

Results A total of 197 pregnancies resulting in 200 live births, $91(45.5 \%)$ female and $109(54.5 \%)$ male exposed to HIV were recruited. The overall MTCT rate was found to be $1.5 \%$ after cessation of all exposures, with two of these babies given mixed-feeding and their mothers not taking anti-retroviral therapy during pregnancy. The factors associated with MTCT in the univariate analysis include HIV diagnosis late during pregnancy, virological suppression \& late ART commencement. Maternal antiretroviral therapy (ART) use \& infant feeding option were found to have significant effect on baby early infant diagnosis (EID) outcome $\left(\chi^{2}=15.40, \mathrm{df}=2, \mathrm{P}=\right.$ $\left.0.001 ; \chi^{2}=12.67, \mathrm{df}=2, \mathrm{P}=0.001\right)$.

Conclusion The diagnosis of HIV and antiretroviral therapy coverage prior to pregnancy are the main factors in the prevention and elimination of mother-to-child transmission of HIV, thus, the achievement of the 90-90-90 goals in HIVinfected women would make it possible to achieve the goal of eliminating perinatal HIV transmission in Nigeria.

Disclosure No significant relationships.

\section{P288 SEXUALLY TRANSMITTED INFECTIONS AMONG PREGNANT WOMEN IN RURAL MYSORE, INDIA}

\footnotetext{
${ }^{1}$ Sandra Kiplagat*, ${ }^{2}$ Makella Coudray, ${ }^{3}$ Karl Krupp, ${ }^{4}$ Kavitha Ravi, ${ }^{4}$ Poornima Jayakrishna, ${ }^{4}$ Vijaya Srinivas, ${ }^{1}$ Purnima Madhivanan. ${ }^{1}$ Florida International University, Stempel College of Public Health - Epidemiology Department, Miami, USA; ${ }^{2}$ Florida International University, Epidemiology, Miami, USA; ${ }^{3}$ Florida International University, Health Promotion and Disease Prevention, Miami, USA; ${ }^{4}$ Public Health Research Institute of India, Mysore, India
}

10.1136/sextrans-2019-sti.409

Background Sexually Transmitted Infections (STIs) including HIV are a major public health problem in India. Syndromic management is the standard of care for STIs, therefore, there are no studies showing the current screening rates for STIs among pregnant women in India. This study examined the burden of STIs among rural pregnant women in Mysore, India.

Methods Between 2009 and 2014, a prospective cohort study was conducted among pregnant women. All women underwent an informed consent process before answering an interviewer-administered questionnaire in the local language of Kannada. The women consented to providing blood and vaginal samples for testing. All participants received routine antenatal care services and were followed-up immediately after delivery, and at 6- and 12-months after delivery. Descriptive, chi square and logistic regression analyses were computed using SPSS 23

Results The mean age of the 1,772 pregnant women were $21.1 \pm 3.2$ years, $98.8 \%$ were Hindu, with $36.4 \%$ belonging to low-income households. The seroprevalence of HIV and Hepatitis B Virus was 0.4\% (95\%CI: 0.1-0.7) and $0.8 \% \quad(95 \%$ CI:0.5-1.3) respectively. There were no cases of Syphilis. The burden of BV was 7.4\% (Nugent Score: 7-10) and 11.6\% for intermediate flora (Nugent score: 4-6). Approximately $8.5 \%$ had any one of the infections (HIV, HBV or BV). STIs were significantly associated with sex under the influence of alcohol (Odds Ratio[OR]: 1.59, 95\%CI: 1.02-2.48) and younger age of sexual initiation (OR:0.90, 95\%CI: 0.83-0.99). Nearly $36.7 \%$ of the infants had low birth weight $(<2.5 \mathrm{~kg})$ and $5.6 \%$ infants died before 28 days. No significant association was observed with STIs and low birth weight and infant deaths.

Conclusion The burden of being diagnosed with any STIs was relatively high at $8.5 \%$ and is associated with risky sexual practices which could inadvertently cause adverse birth outcomes. Therefore, there is an increased need for screening and active intervention targeted to rural pregnant women in India.

Disclosure No significant relationships.

\section{P289 SYPHILIS TESTING AT ANC IN KENYA: DUAL TESTING AS A GAME CHANGER TOWARDS EMTCT}

${ }^{1}$ Carolyne Mati* ${ }^{2}$ Catherine Ngugi, ${ }^{3}$ Rose Wafula, ${ }^{4}$ Bob Agwata, ${ }^{5}$ Kigen Bartilol. ${ }^{1}$ Kenyatta University attached at NASCOP, Student/young Researcher, Nairobi, Kenya; ${ }^{2}$ Ministry of Health, National AIDS and STIS Control Program, Nairobi, Kenya; ${ }^{3}$ UNICEF, EMTCT Specialist Assistant Director of Medical Services (MOH) and EMTCT/POC Specialist, Nairobi, Kenya; ${ }^{4} \mathrm{NASCOP}, \mathrm{MOH}$, Consultant Clinical Pharmacist and Global Fund Operations Manager, Nairobi, Kenya; ${ }^{5} \mathrm{NASCOP}, \mathrm{MOH}$, Head, Nairobi, Kenya

\subsection{6/sextrans-2019-sti.410}

Background The ANC guidelines advocate every woman in the ANC to get tested of both HIV and Syphilis in Kenya. Despite these recommendations, the level of coverage of Syphilis testing has been low, as a result woman are supported to protect their babies from HIV, only for them to lose their babies due to pregnancy loses and babies dying from congenital syphilis. In addition, the HIV negative pregnant women who have undiagnosed and untreated STIs have an increased risk of acquiring HIV. In July 2017, Kenya launched an elimination of MTCT of HIV and Syphilis Strategic Framework 2016-2022. This paved way to the investment and introduction of HIV \& Syphilis Dual test kit use in ANC from March 2018. We thus, sought to know the extent of adherence to syphilis testing among pregnant women at ANC. 
Methods The operational research was a retrospective analysis of reported data from October to December 2016, 2017 and 2018. The data used was sourced from DHIS2.Upon assessment of completeness tabulation was done, and summary statistics obtained.

Results A total of 289,875 women who visited 1stANC in October to December 2016, 277,196 (95.6\%) were tested for HIV and12,161 (4.3\%) tested HIV positive, the proportion that was screened for syphilis was 211,546 (72.9\%) women and out of those,2,396 (1.1\%) women tested positive for syphilis. In October to December 2017, a total of 336,512 women visited ANC, 306,573 (91.1\%) women tested for HIV and 15,056 (4.9\%) turned positive while 262,567 (78\%) were tested for syphilis and 3,072 (1.2\%) turned positive. In 2018 same quarter, 336,687 women visited ANC, 298,598 (88.9\%) were tested for HIV, 16, $5805(6 \%)$ turned positive while 307,842 (91\%) tested for syphilis and 3,464 (1.1\%) tested positive of syphilis. Thus, for the first time ever in Kenya syphilis testing has caught up with HIV testing at $1^{\text {st }}$ ANC. This is an improvement to $91 \%$ from $73 \%$ which is critical for achievements of the country's eMTCT targets.

Conclusion Syphilis not only result $s$ in intrauterine fetal demise, but also facilitates HIV acquisition and impede progress towards eMTCT. Kenya's commitment to reduce MTCT of HIV and Syphilis to below 5\% by 2019 requires our country to improve Syphilis screening/testing. To increase syphilis testing, the use of dual testing kits at ANC should be used widely and every woman at ANC should be encouraged and educated on the importance of syphilis test.

Disclosure No significant relationships.

\section{P290 TEMPORAL DISCOUNTING AND ENGAGEMENT IN CARE AMONG HIV-INFECTED PREGNANT AND BREASTFEEDING WOMEN}

${ }^{1}$ Jessica Londeree Saleska*, ${ }^{2}$ Abigail Norris Turner, ${ }^{3}$ Maria Gallo, ${ }^{1}$ Marcel Yotebieng ${ }^{4}$ Abigail Shoben. ' Ohio State University College of Public Health, Epidemiology, Columbus, USA; ${ }^{2}$ Ohio State University, Internal Medicine, Infectious Diseases, Columbus, USA; ${ }^{3}$ The Ohio State University, Division of Epidemiology, Columbus, USA; ${ }^{4}$ Ohio State University College of Public Health, Biostatistics, Columbus, USA

\subsection{6/sextrans-2019-sti.411}

Background For pregnant or breastfeeding HIV-positive women, poor adherence to antiretroviral (ARV) medication and disengagement from care can increase risk of mother-tochild transmission of HIV. HIV-positive women exhibiting high temporal discounting (TD; defined as the tendency to discount future rewards relative to rewards received closer to the present) may be less likely to adhere to recommendations for the prevention of mother to child transmission (PMTCT).

Methods We performed a secondary analysis of data from a randomized controlled trial conducted in the Democratic Republic of Congo, which assessed the effects of a conditional cash transfer intervention among 433 newly-diagnosed HIVinfected pregnant women. We measured TD at baseline using a delay discounting task. We dichotomized TD (high/low) and examined associations between TD and uptake of available PMTCT services, retention in care and viral suppression at 6 weeks postpartum. We used log-binomial regression models to calculate unadjusted and adjusted risk ratios (RRs) for the effects of high vs. low TD on these outcomes.
Results At baseline, participants had a median age of 29 years, median of 10 years of education, and median gestational age of 26 weeks. The majority (86.6\%) of women exhibited high TD, where $13.4 \%$ exhibited low TD. High TD was associated with incomplete uptake of PMTCT services (adjusted RR: 1.64, 95\% confidence interval (CI): 1.08-2.50) and lower viral suppression at 6 weeks postpartum (adjusted RR: 0.77, 95\% CI: 0.66-0.90). Temporal discounting was not associated with retention to care in unadjusted (RR: 1.57 95\% CI: $0.78-3.15$ ) or adjusted analyses (RR: 1.56 , 95\% CI: $0.76-$ 3.18).

Conclusion Understanding the mechanisms underlying women' behavioral choices is crucial to optimize interventions for behavior change. Our results indicate that women exhibiting high temporal discounting represent a critical population for interventions to improve adherence and engagement in PMTCT care.

Disclosure No significant relationships.

\section{P291 IMPACT OF PMTCT SERVICE UPTAKE ON OUTCOME OF CARE AMONG WOMEN ATTENDING ANTI NATAL CARE IN NIGERIA}

${ }^{1}$ Chidiebere Ezeokafor*, ${ }^{2}$ Adaoha Anosike. ${ }^{1}$ National Agency for the Control of AIDS, Resource Mobilization and Performance Management, ABUJA, Nigeria; ${ }^{2}$ National Agency for the Control of AIDS, Nigeria, Research Monitoring and Evaluation, ABUJA, Nigeria

\subsection{6/sextrans-2019-sti.412}

Background Nigeria has the second largest global burden of HIV/AIDS and also contributes the largest proportion of new vertically acquired HIV infections among children. Despite the effort to control the HIV/AIDS epidemic, elimination of mother to child transmission remains a huge challenge. The study aimed to assess the effect of outcome of care on utilization of prevention of mother to child transmission (PMTCT) services.

Methods A multi stage cross sectional study was conducted in 11 states in Nigeria. A systematic random sampling was used to select 365 clients (women living with HIV) receiving PMTCT services in primary and secondary health facilities. Information on PMTCT and outcome of care were extracted using structured questionnaire while descriptive and bivariate data were analyzed using SPSS version 21.

Results Majority (84.6\%) of the clients received HIV counselling and testing at ANC clinics while 40.2\% were counselled in a group and $26.7 \%$ counselled individually. A high proportion $(93.8 \%)$ of clients were aware of the early infant diagnostic care services being offered while $92.2 \%$ were aware of Navirapin drugs being provided daily for 6 weeks at the health facilities. Similarly, majority $(90.0 \%)$ of the clients were aware that family planning services are provided in the health facilities while $93.8 \%$ had access to infant feeding counselling at ANC. There was no significant difference between outcome of care and utilization of PMTCT services. However, majority of clients who utilized PMTCT services had improved outcome of care.

Conclusion Increased uptake of PMTCT services at ANC contributed to improved outcome of care of pregnant living with HIV and their children. Effort should be made to ensure more women attend ANC to achieve elimination of mother to child transmission in Nigeria.

Disclosure No significant relationships. 